\title{
Analysis of Nonstationary Emissions for Efficient Characterization of Stochastic EM Fields
}

\author{
M H Baharuddin ${ }^{1,2}$, C Smartt ${ }^{1}$, M I Maricar ${ }^{1}$, D W P Thomas ${ }^{1}$, G Gradoni ${ }^{1,3}$, S C Creagh $^{3}$, G Tanner ${ }^{3}$ \\ ${ }^{1}$ The George Green Institute for Electromagnetics Research, University of Nottingham, \\ Nottingham NG7 2RD, UK, E-Mail: Dave.Thomas@nottingham.ac.uk, \\ ${ }^{2}$ Centre of Advanced Electronic and Communication Engineering, Universiti Kebangsaan Malaysia, \\ 43600 Bangi, Malaysia, E-Mail: Mohd.Baharuddin@nottingham.ac.uk, \\ ${ }^{3}$ School of Mathematical Sciences, University of Nottingham, \\ Nottingham NG7 2RD, UK, E-Mail: Gregor.Tanner@nottingham.ac.uk.
}

\begin{abstract}
A statistical approach using field-field correlation functions which is obtained from two-probe time domain measurement is used to characterize the radiation from complex devices. The time-frequency analysis provided by the measurement data has shown that significant emissions may only occur for a few percent of the time and a piecewise stationary model of emissions may be most appropriate. In this paper, a sorting technique is applied to short time segments of the nonstationary time-domain data provided by the measurements. These short time segments are sorted into groups according to the characteristics of the emissions, i.e. different emission processes, with the assumption that the stochastic emissions is stationary within each group (process). The ensemble of time segments associated with each group may then be used to obtain the fieldfield correlations for each process. Results of the analysis give promising insights into how to characterize complex and time dependent systems.
\end{abstract}

Keywords - nonstationary time domain data; radiated emissions; printed circuit boards; piecewise stationary data; time domain measurement; segmentation technique; sorting technique

\section{INTRODUCTION}

Modern electronic devices are becoming more complex, multifunctional and interconnected which can result in unintentional electromagnetic (EM) radiation contaminating the environment and potentially causing interference over a wide frequency of range. Importantly, the emissions processes may be a function of time as well as frequency [1], and it is therefore important to develop a new measurement techniques to characterize such devices. Electromagnetic Interference (EMI) measurement may be carried out in the time-domain as well as in the frequencydomain [2]. Recently, approaches to characterize the stochastic field by performing time domain correlation measurements are described in [1], [3], [4].

A statistical description of EM sources may be used to analyze random sources. An algorithm for propagating the statistical properties of EM fields based on Wigner Functions has been introduced in [5], [6]. This algorithm is used to propagate fieldfield correlation functions from the near-field scanning measurement. The field-field correlation function can be provided through measurement by one-probe measurements [7] and two-probe measurements [3], [8]. For the measurement of complex devices, it is more appropriate to do a two-probe measurement in the time domain because of the lack of phase/time reference. However, two-probe time domain measurements would require a lot of computing power and hard disk space. Other challenges and problems arise from performing the field-field correlation measurements such as the non-stationarity of the measured field discussed in [1], [9], [10].

In order to provide precise input for the propagation algorithm, the field-field correlation function has to be calculated from a stationary emissions source which is sometimes impossible to achieve in real complex systems. It has been discussed in [11] that we should not use methods designed for stationary series on non-stationary series as we will risk getting misleading answer. For example, a process with high emissions may only occur for a small percentage of time, however, the averaging process in calculating the field-field correlation would reduce the influence of this process under the assumption of stationary emissions statistics. To overcome this problem, the nonstationary time series obtained from measurements needs to be segmented into an ensemble of piece-wise stationary time series. Some techniques on the analysis of nonstationary time series discussed in [12] and [13] have been previously implemented in speech processing and for detecting the arrival phases in earthquakes. In [12]-[14] it is shown that a segmentation algorithm can be applied to the nonstationary time series in order to achieve the piecewise-stationary time series.

In this work, a sorting technique is presented in order to characterize and identify the stochastic EM emissions based on the processes or operating modes running on the device. The results will help in the optimization of the near-field scanning process so that devices can be fully characterized as efficiently as possible and worst-case emissions processes may be identified and analysed correctly. This will lead to the development of new and more appropriate standards for emissions testing of complex devices.

\section{ANALYSIS TECHNIQUE OF STOCHASTIC ELECTROMAGNETIC FIELDS}

The field-field propagation algorithm based on Wigner functions [7] requires the input of field-field correlation functions obtained from near field time domain measurements. 
The correlation function $c_{\phi}\left(x_{1}, x_{2}, \tau\right)$ of the field function $\Phi\left(\mathrm{x}_{1}, \mathrm{t}\right)$ is defined as

$$
c_{\Phi}\left(x_{1}, x_{2}, \tau\right)=\lim _{T \rightarrow \infty} \frac{1}{2 T} \int \Phi_{T}\left(x_{1}, t\right) \Phi_{T}\left(x_{2}, t-\tau\right) d t
$$

Here, $\Phi_{T}$ denotes the time windowed field function which is zero for $|\mathrm{t}| \geq \mathrm{T}$. The Fourier Transform of the correlation function gives the correlation spectrum:

$$
\Gamma_{\Phi}\left(x_{1}, x_{2}, \omega\right)=\int_{-\infty}^{\infty} c_{\Phi}\left(x_{1}, x_{2}, \tau\right) \exp (-i \omega t) d t
$$

The correlation matrix may be calculated directly from the frequency domain fields by

$$
\Gamma_{\Phi}\left(x_{1}, x_{2}, \omega\right)=\lim _{T \rightarrow \infty} \frac{1}{2 T}\left\langle\Phi_{T}\left(x_{1}, \omega\right) \Phi_{T}^{*}\left(x_{2}, \omega\right)\right\rangle
$$

where \langle\rangle denotes a suitable ensemble average. The above analysis assumes a stationary process; however, for complex devices this assumption may not be valid. In this case a piecewise stationary assumption may be justifiable in which case the analysis should be performed separately for each of the emissions processes occurring. This indicates that elements of the ensemble must be grouped together or sorted according to their statistics.

The sorting process is proposed to be implemented on each segments of the time-domain data of the stochastic EM emissions to test whether they are produce by the same operating modes of the device under test or not. This test is similar to a Kolmogorov Smirnov (K-S) two sample test [15] which looks into the greatest distance between two empirical distribution function (ECDF) from two samples under test. The $\mathrm{K}-\mathrm{S}$ test is performed to decide if two samples come from the same distribution. But instead of using ECDF, in applying this test to the stochastic EM emissions, we find it to be more appropriate to look into the greatest distance between two cumulative spectral density functions of two samples. The cumulative spectral density function for both samples are defined as:

$$
\begin{gathered}
P_{1}(x)=\frac{\int_{f_{1}}^{f}\left|X_{1}\left(f^{\prime}\right)\right| d f^{\prime}}{\int_{f_{1}}^{f_{2}}\left|X_{1}\left(f^{\prime}\right)\right| d f^{\prime}} \\
P_{2}(x)=\frac{\int_{f_{1}}^{f}\left|X_{2}\left(f^{\prime}\right)\right| d f^{\prime}}{\int_{f_{1}}^{f_{2}}\left|X_{2}\left(f^{\prime}\right)\right| d f^{\prime}}
\end{gathered}
$$

where $X_{1}\left(f^{\prime}\right)$ and $X_{2}\left(f^{\prime}\right)$ are the field spectrum for the first and second segments respectively. Then we compare the two cumulative spectral density functions as follows:

$$
\begin{gathered}
D_{1,2}=\max \left(\left|P_{1}(x)-P_{2}(x)\right|\right) \\
D_{a}=\text { Threshold value }
\end{gathered}
$$

The null hypothesis is rejected if $D_{1,2}>D_{a}$. Fig. 1 shows the illustration of how the sample data is tested. Two field spectra $X_{1}$ (blue) and $X_{2}$ (orange) are shown in Fig. 1(a). Then
(4) and (5) is used to produce the cumulative spectral density function plots shown in Fig. 1(b) for both data. Equation (6) is then used to check the greatest distance between those two plots which is going to be compared against $D_{a}$.

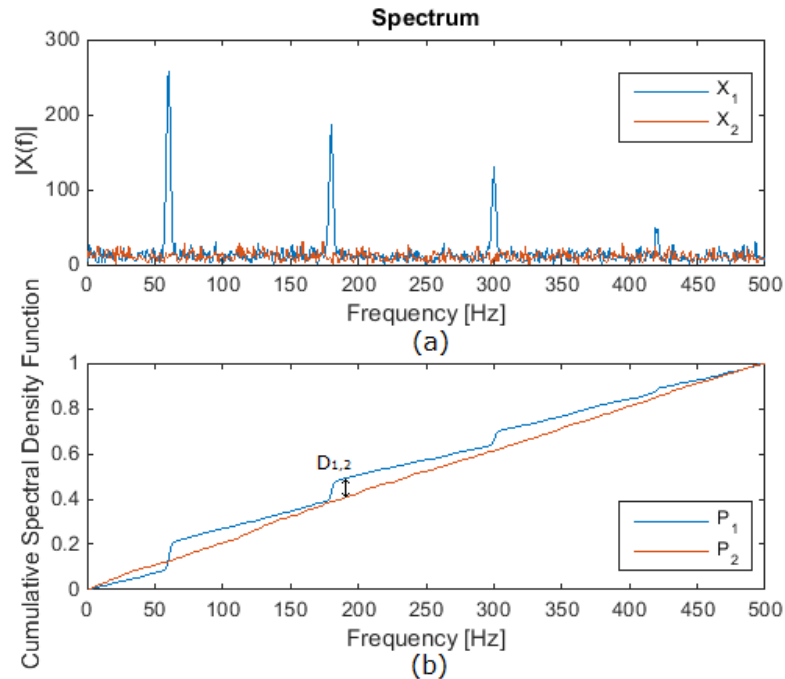

Fig. 1 Illustration of two-segment comparison

In this initial implementation of sorting technique on the EM emissions, we aim to identify emissions that are related to the processes running based on the broadband response produced. This analysis technique should allow us to post process the data for each emissions process separately and then provide the necessary information to be processed by the propagation algorithm.

\section{MEASUREMENT PROCESS OF NONSTATIONARY EMISSIONS FROM A COMPLEX DEVICE}

The measured correlation data described in [3] are suitable for validation of the statistical field propagation techniques. However, the analysis of real world systems provides significant challenges as we cannot necessarily obtain stationary emissions that need to be applied to (3) so as to predict their propagation. In real applications, most of the stochastic EM emissions are nonstationary which means that they are time dependent and only occur for a very short period of time. An important consideration in the characterization of a complex PCB is what processes should be running on the board whilst the measurement is taking place. We believe that processes running on the PCB will determine the stationarity of the EM emissions [1].

Intel Galileo [16] is a microcontroller board consists of compact integrated circuits which can be programmed to run a specific task in an embedded system. We consider that the way the microcontroller is designed which includes memory, programmable input/output peripherals and processor to be a good example of a realistic complex source for this measurement. To demonstrate the issue of nonstationarity of the EM emissions, two programs have been designed as follows:

Program 1 - A large array was allocated and random elements were filled with random numbers followed by blinking LED 
Program 2 - A large array was allocated and random elements were filled with random numbers.

Each program runs in a loop once the Galileo is switched ON. The selection of the program running is controlled by a switch connected to the Galileo. The difference between those two programs is that in Program 1 there are two processes (filling random number into random elements and LED blinking) running onboard while in Program 2, there is only one process (filling of random numbers into random elements) running onboard.

Two sample data have been captured while the Galileo is running Program 1 and Program 2 to look at the measured field. A Langer EMV-Technik RF R50-1 magnetic field probe is attached to a stand and positioned at a height of $2 \mathrm{~mm}$ above the memory chip on the Galileo as depicted in Fig. 2. The probe is then connected to channel 1 of an $8 \mathrm{GHz}$ KEYSIGHT DSOS804a Digital Oscilloscope which is capable of capturing data from the probe with $20 \mathrm{GSa} / \mathrm{s}$ max sample rate and 50 Mpts/channel of standard memory. The time domain sampling rate is set to be $2 \mathrm{Gsa} / \mathrm{s}$ and two sets of 2,097,152 time samples were recorded while Program 1 and Program 2 are running on the Galileo.

The time-domain plot from the emissions of a Galileo running Program 1 and its corresponding time-frequency plot are shown in Fig. 3 and Fig. 4 respectively while a time-domain plot for the emissions while running Program 2 and its corresponding time-frequency plot are shown in Fig. 5 and Fig. 6 respectively.

In Fig. 4, we can see that the measured field is not stationary where its characteristics are a function of time. Much of the field spectrum is constant over time however the plot does show an event which produces a broad band response which is present for only a few percent of the total observed time. We observed that with Program 2 running on the Galileo we obtained quite a consistent field spectrum over time. This can be clearly seen in Fig. 6.

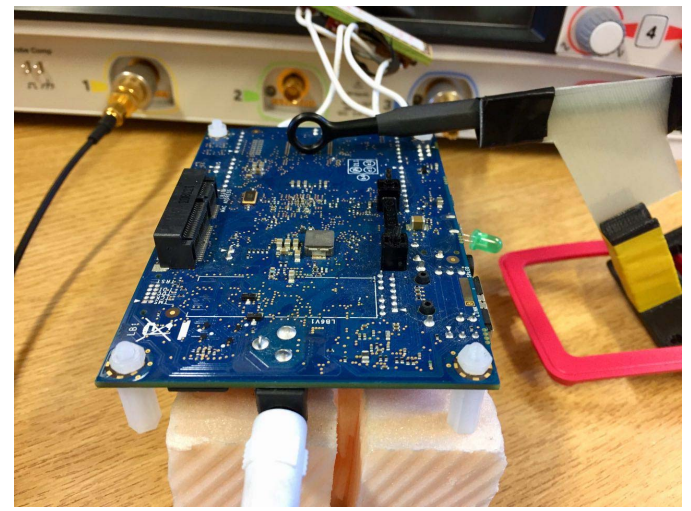

Fig. 2 Intel Galileo

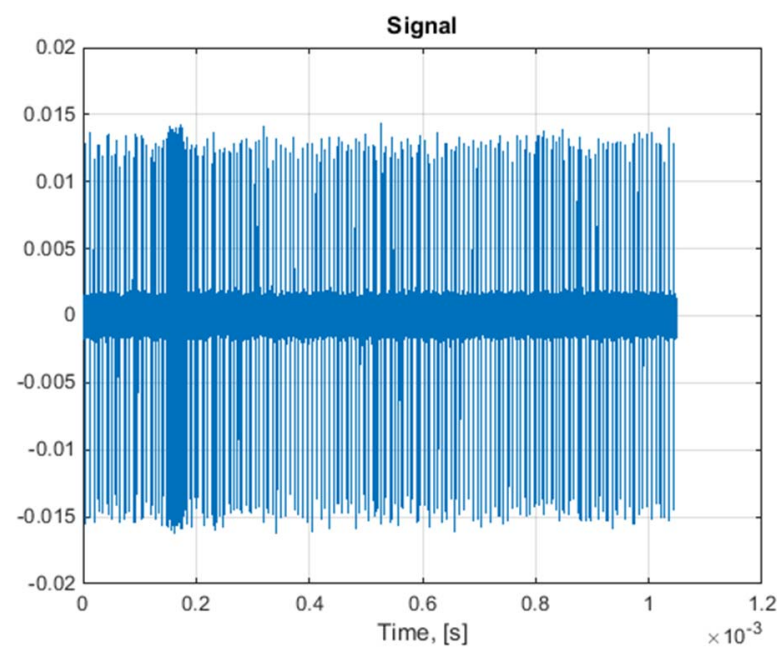

Fig. 3 Time-series for Galileo running Program 1

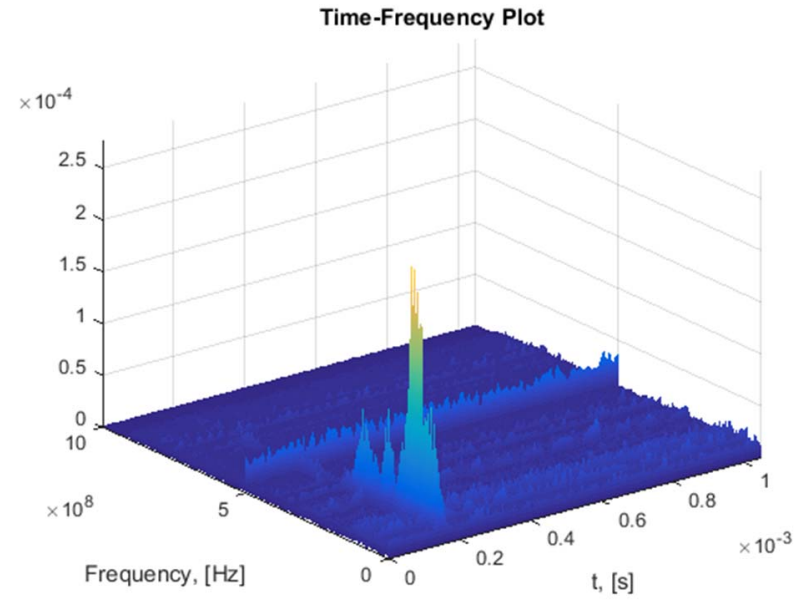

Fig. 4 Time-Frequency plot for Galileo running Program 1

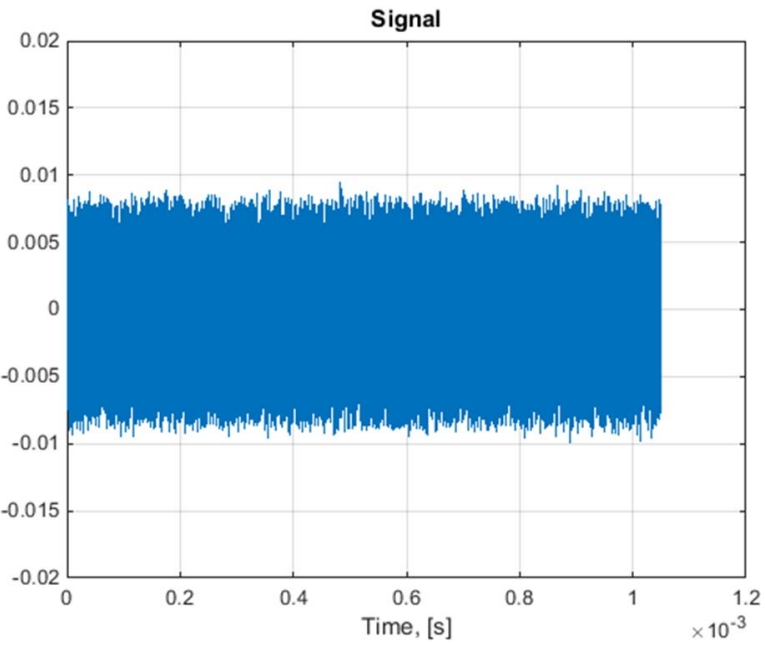

Fig. 5 Time-series for Galileo running Program 2 


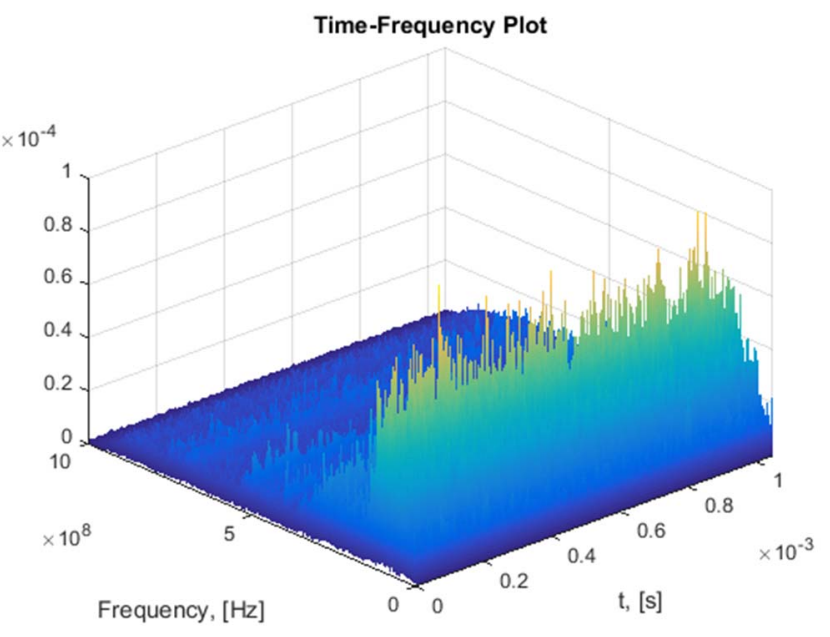

Fig. 6 Time-Frequency plot for Galileo running Program 2

\section{RESULTS}

This process sorting technique aims to investigate the possibility of dealing with nonstationary emissions from the measurement and sort the datasets based on processes running on the Galileo at a given time. In this example, the nonstationary time domain dataset which is shown in Fig. 3 is used to illustrate the application of the proposed technique. However, for this early investigation on the sorting of nonstationary stochastic EM emissions, we fixed the number of segments to 16 to focus more on the feasibility of the proposed technique. Each sub-segment of the full-time domain dataset captured will have $2^{17}=131,072$ time samples which we assume to be stationary within each sub-segment. This division into sub-segments is shown in Fig. 7. The Fourier Transform of each of these datasets will produce the frequency domain datasets which will be applied to (4) and (5).

Considering the broadband response in Fig. 4, we are interested in looking at the measured field between $50-150 \mathrm{MHz}$ so the test will be applied on the measured field between those frequencies. Equation (6) is used to look at the maximum distance between the cumulative sums of the magnitude of the field spectrum between two adjacent points. If the distance is less than the threshold value, they will be grouped together and the test continue until all the segments have been compared.

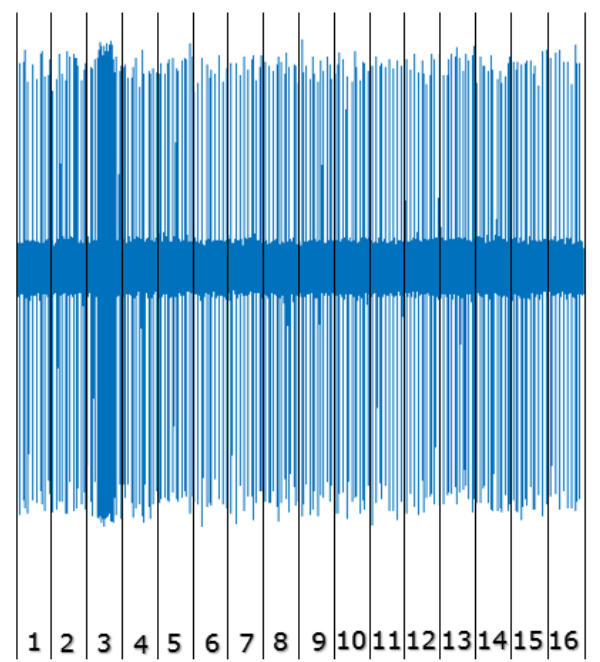

Fig. 7 Time-series from the measurement are divided into 16 segments

From the result of the test, two different processes are identified which are Process 1 that we believe to occur when the LED is blinking and Process 2 when the memory intensive program (filling random number into random elements) is running. Out of 16 shorter time domain segments, 15 segments are produced while the Galileo is running Process 1 and only one segment while running Process 2 as can be seen in Fig. 8 and Fig. 9 respectively.

Process 1 - number of segments: 15

Signal
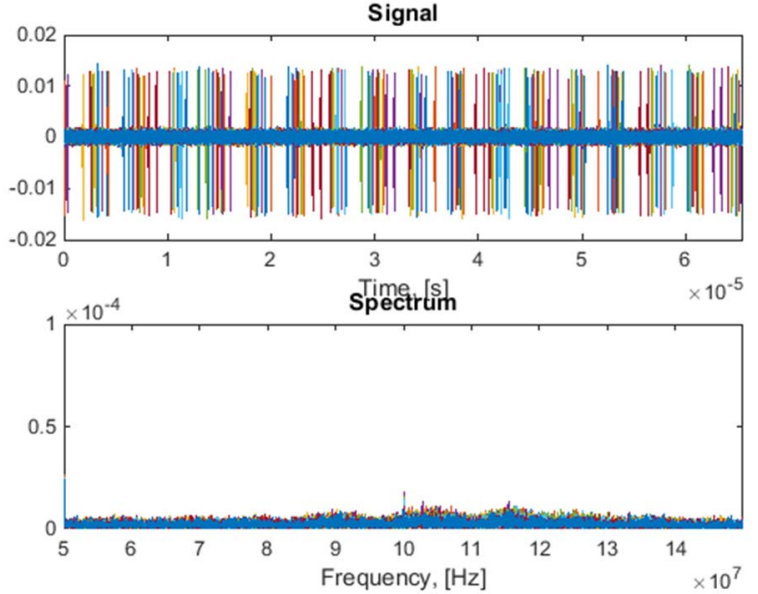

Fig. 8 Compilation of shorter time-domain datasets when process 1 is running on Galileo 
Process 2 - number of segments: 1 Signal
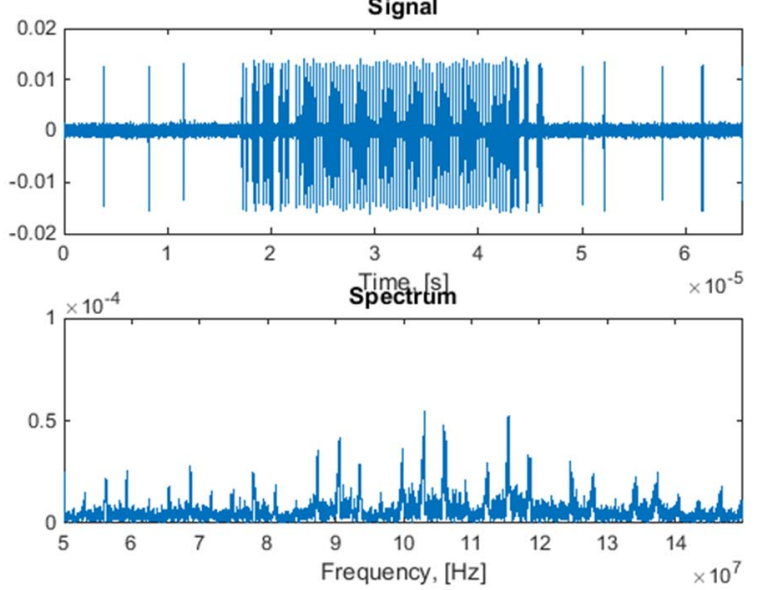

Fig. 9 Compilation of shorter time-domain datasets when process 2 is running on Galileo

We can clearly see that the field spectrum produced when process 2 is running has a very different character compare to when process 1 is running. From the test we could also determine on which segments the processes occur. A plot to show the occurrence of the processes against the segment number is produced and shown in Fig. 10. The test accurately indicates that process 2 occurs at the third segment and the rest of the segments are associated with process 1 .

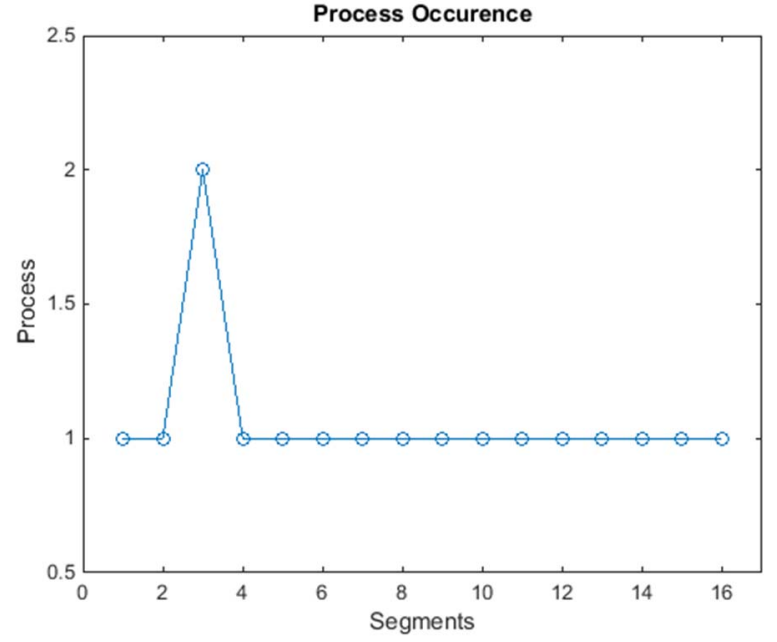

Fig. 10 Occurrence of Process 1 and 2 against segments number

\section{CONCLUSIONS}

In this paper, we are proposing a new approach on how to characterize radiation from a complex and time dependent system with non-stationary emissions. We have highlighted the importance of handling the non-stationary measured field before applying propagation algorithms for stationary random fields. A method has been described which sorts short-time segments of near field time domain data into a number of ensembles of datasets with stationary statistics within each ensemble (i.e. a piecewise stationary model). This method has been found to work very well for a DUT with two known processes running on the device. The advantage of this technique is that worst case emissions processes may be readily identified and their full impact assessed, including their possibly impulsive nature. Without application of this sort of technique the predicted impact of short duration emissions processes is likely to be reduced by the effect of averaging within statistically based analysis methods.

At this point, no priori knowledge of the processor is needed to determine the number of segments but estimation is currently been made based on the estimated percentage of the change in emissions over the whole time domain period. Improvements to the methodology presented can be envisaged in which the segmentation procedure determines the change points in the nonstationary time domain data rather than simply sorting subsets of data (the problem with this is that the process can change part way through a dataset).

One of the issues in performing a two-probe time domain measurement is that a full measurement will produce a large amount of data and it will be quite a challenge to process it. It is hoped that the technique outline in this paper can be used to overcome this problem by implementing the sorting process at the measurement stage. By doing so, only the essential data will be stored, ensuring that sufficient data is obtained for each emissions process of interest, without requiring to store excessive amount of data. Work is ongoing in introducing this method to the full two-probe measurement to see if it is efficient enough to handle the thousands of measurements needed.

\section{ACKNOWLEDGMENT}

The authors would like to acknowledge the support of the European Commision under the FETopen program NEMF21 and Universiti Kebangsaan Malaysia, 43600 UKM Bangi, Selangor, Malaysia.

\section{REFERENCES}

[1] C. Smartt et al., "Challenges of time domain measurement of fieldfield correlation for complex PCBs," in 2015 IEEE International Symposium on Electromagnetic Compatibility (EMC), 2015, pp. 953-958.

[2] P. Russer, "EMC measurements in the time-domain," in 2011 XXXth URSI General Assembly and Scientific Symposium, 2011, pp. 1-35.

[3] M. H. Baharuddin et al., "Measurement and Wigner function analysis of field-field correlation for complex PCBs in near field," in 2016 International Symposium on Electromagnetic Compatibility - EMC EUROPE, 2016, pp. 7-11.

[4] J. A. Russer and P. Russer, "An efficient method for computer aided analysis of noisy electromagnetic fields," in 2011 IEEE MTT-S International Microwave Symposium, 2011, pp. 1-4.

[5] G. Gradoni, S. C. Creagh, and G. Tanner, "A wigner function approach for describing the radiation of complex sources," IEEE Int. Symp. Electromagn. Compat., vol. 2014-Septe, no. September, pp. 882-887, 2014.

[6] G. Gradoni, S. C. Creagh, and G. Tanner, "A phase-space approach for propagating field-field correlation functions near stochastic sources," in 2016 URSI International Symposium on Electromagnetic 
Theory (EMTS), 2016, pp. 678-681.

[7] G. Gradoni et al., "Wigner-Function-Based Propagation of Stochastic Field Emissions From Planar Electromagnetic Sources," IEEE Trans. Electromagn. Compat., vol. 60, no. 3, pp. 580-588, Jun. 2018.

[8] G. Gradoni et al., "Near-Field Scanning and Propagation of Correlated Low-Frequency Radiated Emissions," IEEE Trans. Electromagn. Compat., pp. 1-4, 2017.

[9] D. W. P. Thomas et al., "Near-field scanning of stochastic fields considering reduction of complexity," in 2017 International Symposium on Electromagnetic Compatibility - EMC EUROPE, 2017, pp. 1-6.

[10] D. W. P. Thomas, M. H. Baharuddin, C. Smartt, G. Gradoni, G. Tanner, and S. Creagh, "Reducing the complexity of near-field scanning of stochastic fields," in 2017 13th International Conference on Advanced Technologies, Systems and Services in Telecommunications (TELSIKS), 2017, pp. 11-14.

[11] G. P. Nason, H. M. Mader, S. G. Coles, C. B. Connor, and L. J. Connor, "Stationary and Non-stationary Time Series," Chapter 11 Stat. Volcanol., no. Geological Society for IAVCEI, UK, 2006.

[12] M. Last and R. Shumway, "Detecting abrupt changes in a piecewise locally stationary time series," J. Multivar. Anal., vol. 99, no. 2, pp. 191-214, Feb. 2008.

[13] S. Adak, "Time-Dependent Spectral Analysis of Nonstationary Time Series," J. Am. Stat. Assoc., vol. 93, no. 444, pp. 1488-1501, Dec. 1998.

[14] A. Ramachandra Rao, K. H. Hamed, and H.-L. Chen, "Segmentation of Non-Stationary Time Series," in Nonstationarities in Hydrologic and Environmental Time Series. Water Science and Technology Library, vol 45., Springer, Dordrecht, 2003, pp. 213-252.

[15] N. I. of S. and Technology, "KOLMOGOROV SMIRNOV TWO SAMPLE," $2016 . \quad$ [Online]. Available: http://www.itl.nist.gov/div898/software/dataplot/refman1/auxillar/ks 2samp.htm.

[16] Arduino, “Intel Galileo," 2018. [Online]. Available: https://www.arduino.cc/en/ArduinoCertified/IntelGalileo. 